\title{
Antibiotic Stewardship in Premature Infants: A Systematic Review
}

\author{
Polona Rajar ${ }^{a, b}$ Ola D. Saugstad ${ }^{c}$ Dag Berild ${ }^{d, e} \quad$ Anirban Dutta $^{f}$ \\ Gorm Greisen $^{g} \quad$ Ulrik Lausten-Thomsen $^{g}$ Sharmila S. Mande ${ }^{f}$ Sushma Nangia ${ }^{\text {h }}$ \\ Fernanda C. Petersen ${ }^{b}$ UIf R. Dahle ${ }^{i}$ Kirsti Haaland ${ }^{a}$ \\ ${ }^{a}$ Department of Paediatrics, Oslo University Hospital Ullevål, Oslo, Norway; ${ }^{b}$ Institute of Oral Biology, University of \\ Oslo, Oslo, Norway; ${ }^{\circ}$ Department of Paediatric Research, University of Oslo, Oslo, Norway; ${ }^{\mathrm{d} D e p a r t m e n t}$ of Infectious \\ Diseases, Oslo University Hospital, Oslo, Norway; Institute of Clinical Medicine, Faculty of Medicine, Oslo University, \\ Oslo, Norway; fTCS Research, Tata Consultancy Services Ltd, Pune, India; ${ }^{9}$ Department of Neonatology, Copenhagen \\ University Hospital Rigshospitalet, Copenhagen, Denmark; ${ }^{h}$ Lady Hardinge Medical College and Kalawati Saran \\ Hospital, New Delhi, India; 'Centre for Antimicrobial Resistance, Norwegian Institute of Public Health, Oslo, Norway
}

\section{Keywords}

Antibiotic stewardship · Premature infant · Antibiotic resistance

\begin{abstract}
Introduction: Antibiotic treatment in premature infants is often empirically prescribed, and practice varies widely among otherwise comparable neonatal intensive care units. Unnecessary and prolonged antibiotic treatment is documented in numerous studies. Recent research shows serious side effects and suggests long-term adverse health effects in prematurely born infants exposed to antibiotics in early life. One preventive measure to reduce unnecessary antibiotic exposure is implementation of antibiotic stewardship programs. Our objective was to review the literature on implemented antibiotic stewardship programs including premature infants with gestational age $\leq 34$ weeks. Methods: Six academic databases (PubMed [Medline], McMaster PLUS, Cochrane Database of Systematic Reviews, UpToDate, Co-
\end{abstract}

chrane Central Register of Controlled Trials, and National Institute for Health and Care Excellence) were systematically searched. PRISMA guidelines were applied. Results: The search retrieved 1,212 titles of which 12 fitted inclusion criteria (11 observational studies and 1 randomized clinical trial). Included articles were critically appraised. We grouped the articles according to common area of implemented stewardship actions: (1) focus on reducing initiation of antibiotic therapy, (2) focus on shortening duration of antibiotic therapy, (3) various organizational stewardship implementations. The heterogeneity of cohort composition, of implemented actions and of outcome measures made meta-analysis inappropriate. We provide an overview of the reduction in antibiotic use achieved. Conclusion: Antibiotic stewardship programs can be effective for premature newborns especially when multifactorial and tailored to this population, focusing on reducing initiation or on shortening the duration of antibiotic therapy. Programs without specific measures were less effective.

(c) 2020 The Author(s)

Published by S. Karger AG, Basel karger@karger.com www.karger.com/neo

Karger $\stackrel{\text { ' }}{5}$

BOPEN ACCESS
(C) 2020 The Author(s)

Published by S. Karger AG, Basel

This is an Open Access article licensed under the Creative Commons Attribution-NonCommercial-4.0 International License (CC BY-NC) (http://www.karger.com/Services/OpenAccessLicense), applicable to the online version of the article only. Usage and distribution for commercial purposes requires written permission.
Kirsti Haaland

Department of Paediatrics, Oslo University Hospital Ullevål

Kirkeveien 166

Oslo 0450 (Norway)

uxkila@ous-hf.no 


\section{Introduction}

Treatment and survival of newborn infants, in particular the premature, often rely on effective antibiotics. Infections are leading causes of morbidity in infancy, contributing to $15 \%$ of neonatal deaths worldwide (2017) [1]. Incidence and mortality rates of early-onset sepsis (EOS) are inversely proportional to gestational age (GA) and birth weight [2]. Early neonatal sepsis is often defined by positive microbial cultures from blood or cerebrospinal fluid (obtained within $72 \mathrm{~h}$ after birth, and late-onset sepsis after $72 \mathrm{~h}$ ), in patients with signs or symptoms of systemic infection [2,3]. Blood cultures are, however, often falsely negative due to difficulties in obtaining sufficient volume, low bacteremia levels, and intrapartum antibiotics [4]. Also, results are not ready before necessary decision on initiation of antibiotics. As laboratory tests may be unspecific and delayed, and clinical signs can be prone to subjective interpretation, risk assessment is often used, with a low threshold for starting empiric antibiotic therapy [2].

Uncertain clinical symptoms and signs, potential disastrous outcome in case of delayed start of antibiotic treatment, and reluctance to withdraw initiated treatment often result in overuse of antibiotics in the neonatal intensive care unit (NICU). In premature infants, antibiotic treatment for $>5$ days in infants with negative blood cultures is associated with increased risk of necrotizing enterocolitis, bronchopulmonary dysplasia, invasive fungal infections, retinopathy, periventricular white matter damage, and death [5-8]. In addition, antibiotic disruption of the developing microbiome may carry lasting consequences reflected as dysbiosis and increased carriage of antibiotic resistance genes and multidrug resistant organisms $[9,10]$.

An antibiotic stewardship program (ASP) is defined as "ongoing efforts by a health care organization to optimize antimicrobial use among hospitalized patients in order to improve patient outcomes, ensure cost-effective therapy, and reduce adverse sequelae of antimicrobial use (including antimicrobial resistance)" [11]. Battles against drugresistant organisms are becoming increasingly challenging and implementation of ASPs is rightfully increasing $[12,13]$. For premature infants, the main focus of ASPs entails reducing empiric antibiotics after birth and restricting duration of antibiotic therapy in low risk situations. Additional focus areas include antibiotics pre- and intrapartum, drug selection, dosage, and more $[2,14,15]$. In addition to ASPs, infection prevention and control actions (from hand hygiene, visitor limitations, sterile equipment, and vaccination of health care workers, to interventions related to infrastructure, number of health care workers, and special isolation actions) result in lower incidence of healthcare-related infections and thus lower antibiotic prescription rates [16]. ASPs implemented alongside infection prevention and control are more successful than when implemented alone [17].

The risk-based approach with low threshold is often used for starting antibiotics right after birth, an approach that has successfully lowered EOS incidence but increased number of noninfected infants exposed to antibiotics [4, 18]. Such empiric therapy is often extended to 5 to 7 days even in the absence of positive blood cultures [19]. In a recent study, Flannery et al. [20] demonstrated that the majority of infants $<1,500 \mathrm{~g}$ from nearly 300 US hospitals were treated with antibiotics in their first days of life, and proximately $1 / 3$ received $>5$ days of antibiotic treatment. There were major differences between hospitals that could not be explained solely by medical reasons. In the period from 2015 to $2018>50 \%$ of infants born at GA $<32$ weeks received intravenous antibiotics within the first 14 days of life [21]. Median treatment duration (interquartile range) was 8 (7-10) and 6 (5-7) days for culture-positive and culture-negative EOS, respectively, in the period from 2009 to 2011, and there was great interhospital variation (Norwegian Neonatal Network database) [21].

Antibiotics are essential drugs and their use should be expected to remain high in premature infants, but unnecessary antibiotic exposure must be minimized due to substantial risks of adverse effects [22]. This review aims to summarize available knowledge on ASPs implemented for infants born before 34 weeks GA.

\section{Methods}

This systematic review was performed using all applicable items from the PRISMA guidelines (see online suppl. file 1; see www.karger.com/doi/10.1159/000511710 for all online suppl. material).

We performed a search on July 9, 2019, in 6 academic databases. Additional 10 articles were obtained from reference lists. Full search terms and search strategy are provided (online suppl. file 2). A second search performed on December 5, 2019, revealed no additional studies. No previous systematic review of ASPs in premature infants was identified.

We retrieved 1,212 titles, and no duplicates were found. Three authors (P.R., O.D.S., and U.R.D.) screened the titles and abstracts of all $(1,212)$ articles were identified through the search. Comments and guidelines were excluded. We included articles that incorporated any premature infants born $\leq 34$ weeks GA. Infants with extremely low birth weight $(<1,000 \mathrm{~g})$ and very low birth weight $(<1,500 \mathrm{~g})$ were also regarded as born $\leq 34$ weeks GA where 


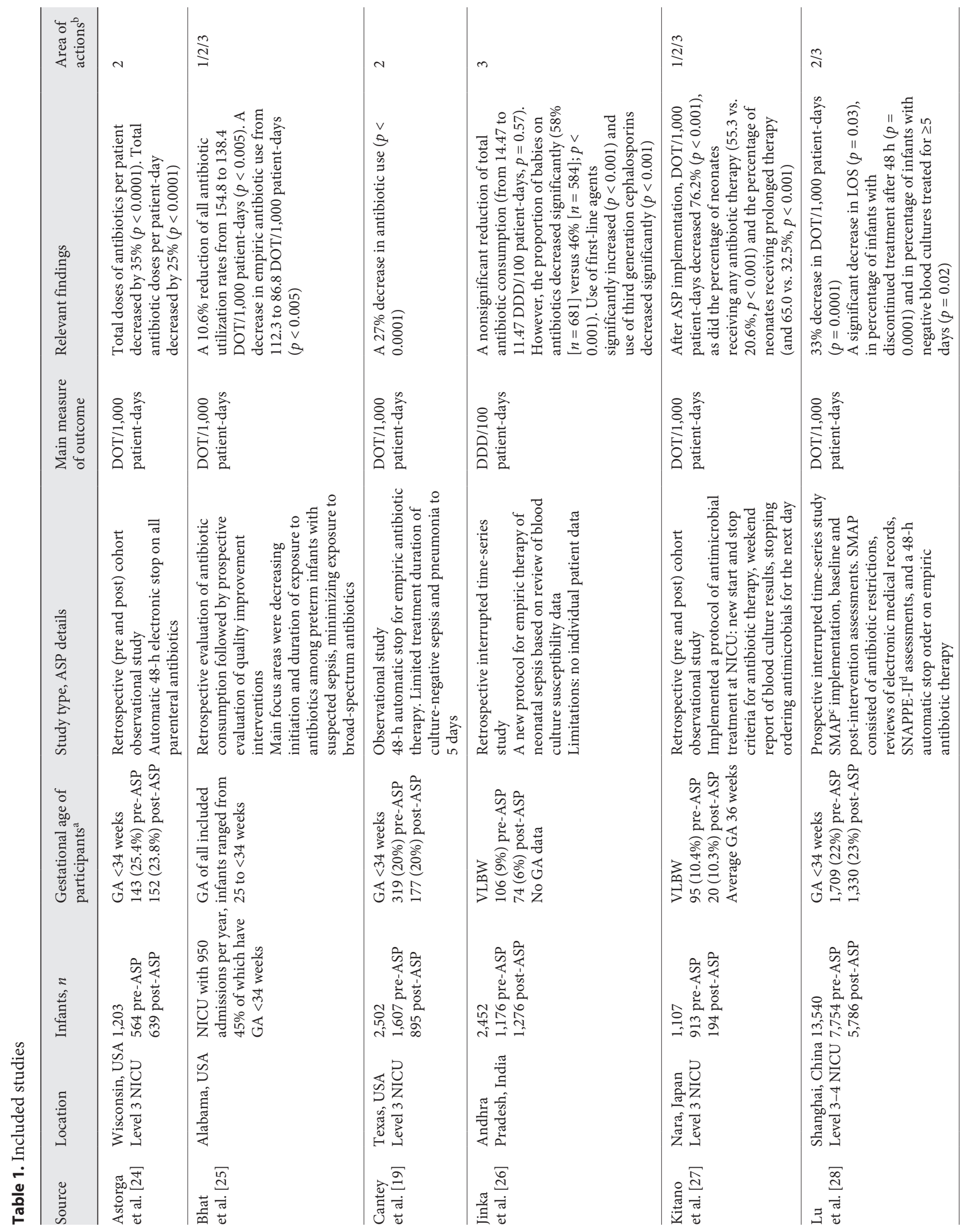




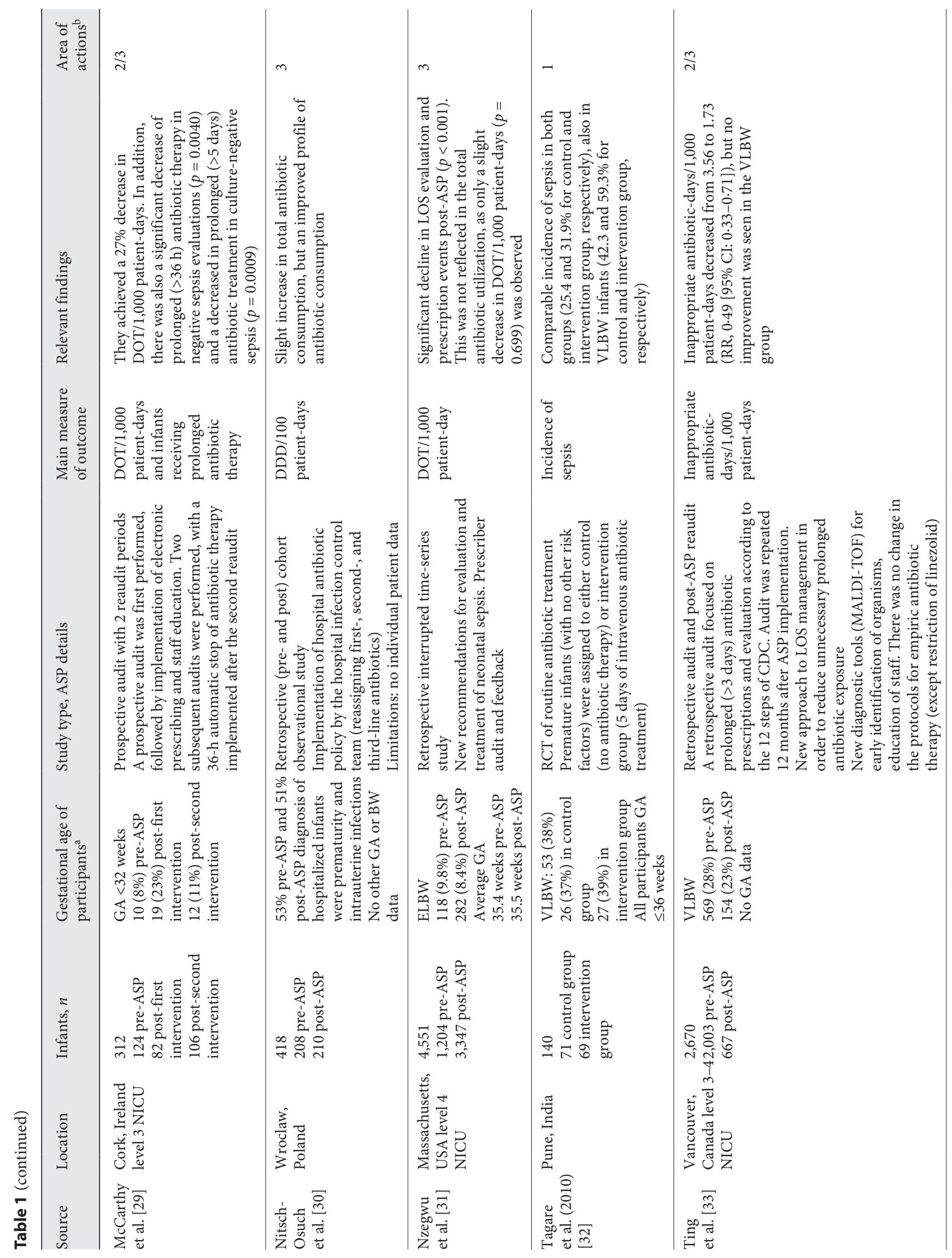




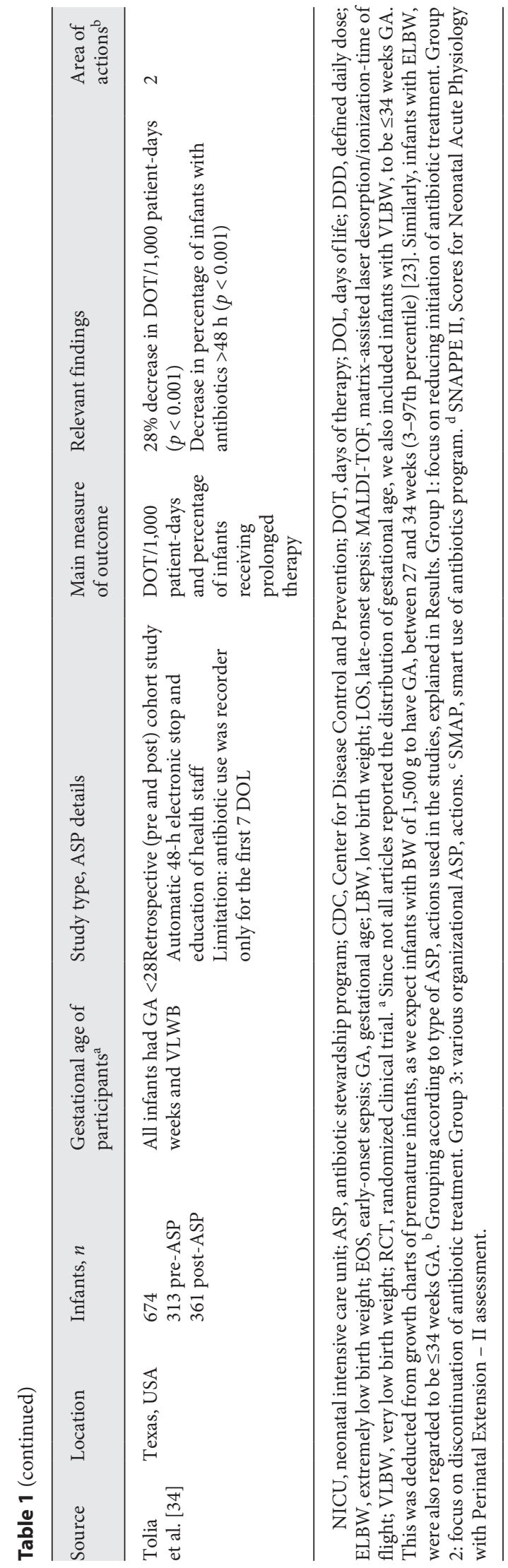

Antibiotic Stewardship in Premature Infants
GA of included infants was not transparent from the article [23]. We included articles that compared use of antibiotics before and after ASP implementation. We excluded articles where antibiotic stewardship actions were directed toward specific microorganisms and articles that reported the current state of antibiotic consumption and possibilities for ASP but lacked results of ASP actions. Papers that targeted antibiotic usage in a more specific group of infants only (e.g., surgical prophylaxis) were also excluded. In total, 29 full-text articles were retrieved for these criteria, or required more information than was provided in the abstract for an informed decision. Two investigators (P.R. and K.H.) independently assessed the full-text articles. A total of 12 articles were included in the review (Table 1; Fig. 1). Seventeen studies were excluded for reasons described in Table 2 . Study quality and risk of bias were assessed by 2 investigators (P.R. and K.H.), using Newcastle-Ottawa quality assessment scale for the 11 cohort studies and Jadad scale for the randomized clinical trial (RCT) (online suppl. 3, 4).

\section{Results}

The 12 selected articles varied greatly in their study population, interventions, and outcome measures (as detailed in Table 1). To summarize and compare their findings we identified (1) common areas of action (Fig. 2) and (2) common units of measurement for reporting results (Fig. 3, 4). Five articles included more than one area of action.

\section{Common Areas of Action (3 Groups)}

Group 1: three out of the 12 studies focused on restricting initiation of antibiotics. Tagare et al. [32] performed a RCT to evaluate the protective effect of empiric antibiotic coverage in premature infants in low-risk situations. Infants with no other risk for infections were randomized to the control or to the intervention group with 5 days of antibiotic prophylaxis. Bhat et al. [25] encouraged empirical antibiotic use only in the presence of perinatal risk factors for EOS or in infants with postnatal clinical illness suggestive of evolving sepsis. Kitano et al. [27] implemented comprehensive criteria for initiation of antibiotic treatment, based on maternal chorioamnionitis, infant's clinical presentation, and laboratory values combined. Both Kitano et al. and Bhat et al. [25, 27] also applied interventions from the 2 other areas of action.

Group 2: eight out of the 12 studies implemented actions toward reducing duration of antibiotics. Astorga et al. [24] implemented a 48-h automatic stop on empiric antibiotics initiated in infants at risk for infection without other changes to their practice. The same was done by Cantey et al. [19], additionally limiting treatment duration of culture-negative sepsis and pneumonia to 5 days. 


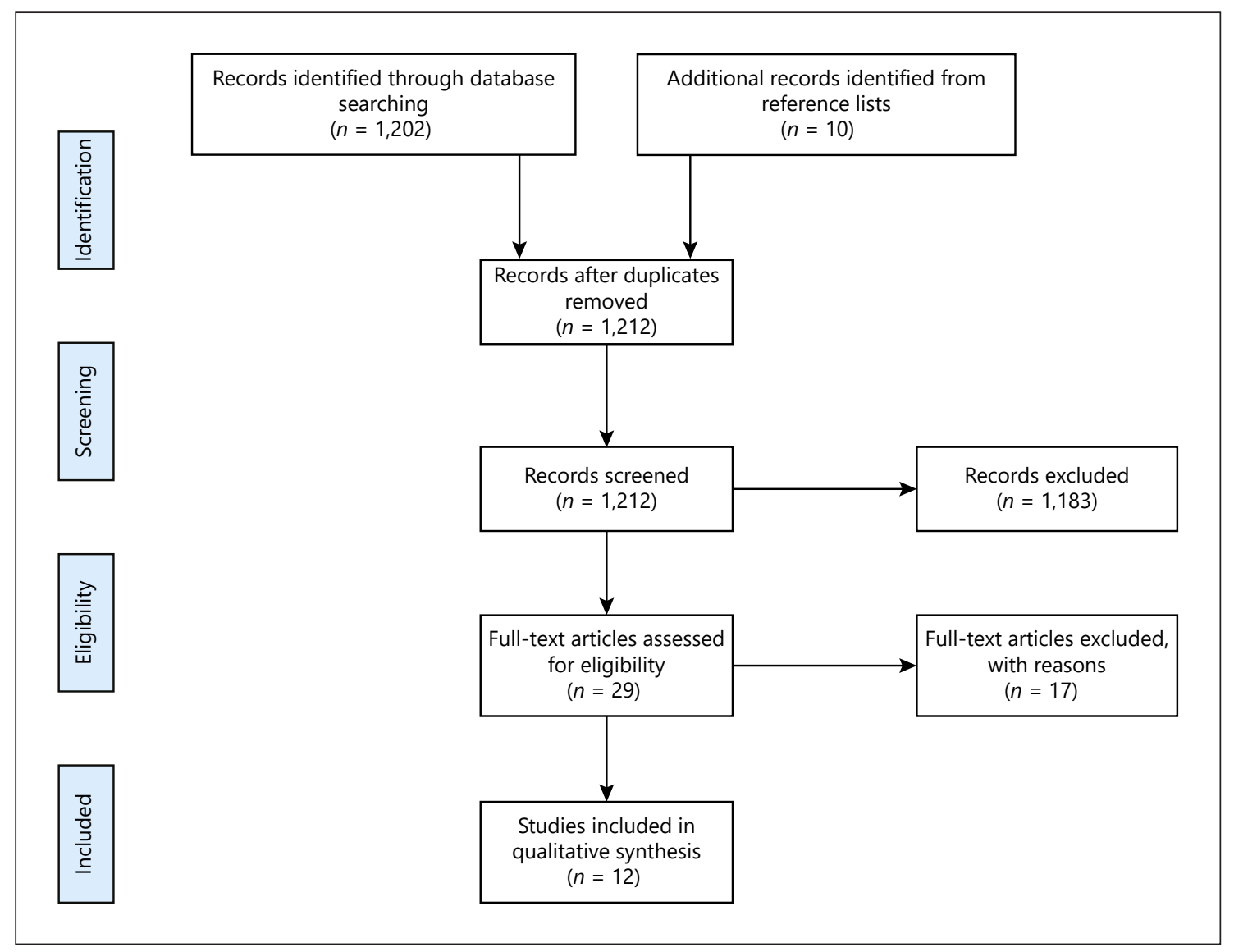

Fig. 1. Study selection process.

Tolia et al. and Lu et al. $[28,34]$ implemented an automatic stop order at $48 \mathrm{~h}$ in addition to other educational and organizational ASP actions, respectively. McCarthy et al. [29] specifically targeted prolonged antibiotic courses in their second of 2 intervention periods, implementing an automatic stop after $36 \mathrm{~h}$ in asymptomatic infants with 2 negative CRP and negative blood culture. Similarly, 3 more studies encouraged discontinuation of antibiotic treatment within $36-48 \mathrm{~h}$ in infants with negative cultures and no clinical or laboratory suspicion of sepsis $[25,27,33]$.

Group 3: eight out of the 12 studies implemented various organizational ASP actions. Jinka et al. and NitschOsuch et al. [26, 30] implemented a protocol for empiric treatment and for antibiotic prescriptions, respectively. They provided no individual level data. Nzegwu et al. [31] evaluated the implementation of new guidelines for neonatal infection assessment and unit-wide ASP education, focusing especially on management of late-onset sepsis, without any specific actions (such as an automatic stop order) taken. The remaining 5 studies used also actions from groups 1 or 2, described above. Lu et al. [28] reassigned first, second, and third line antibiotic to restrict consumption of broad-spectrum antibiotics. They reviewed electronic records of all antibiotic use in the NICU. Health personnel was informed and trained for the ASP interventions after the baseline period. McCarthy et al. [29] also focused on educational interventions based on monitored antibiotic prescribing data. Ting et al. [33] implemented 3 of the 12 -steps program from Centers for Disease Control and Prevention, adjusted for the NICU population: "target the pathogen," "practice antimicrobial control," and "know when to say no." After performing a retrospective audit for prolonged ( $>3$ days) antibiotic prescriptions, their multidisciplinary ASP NICU team defined appropriate uses of antibiotics in different clinical situations, performed staff education, and implemented a new diagnostic tool to allow for earlier 
Table 2. Full-text articles excluded

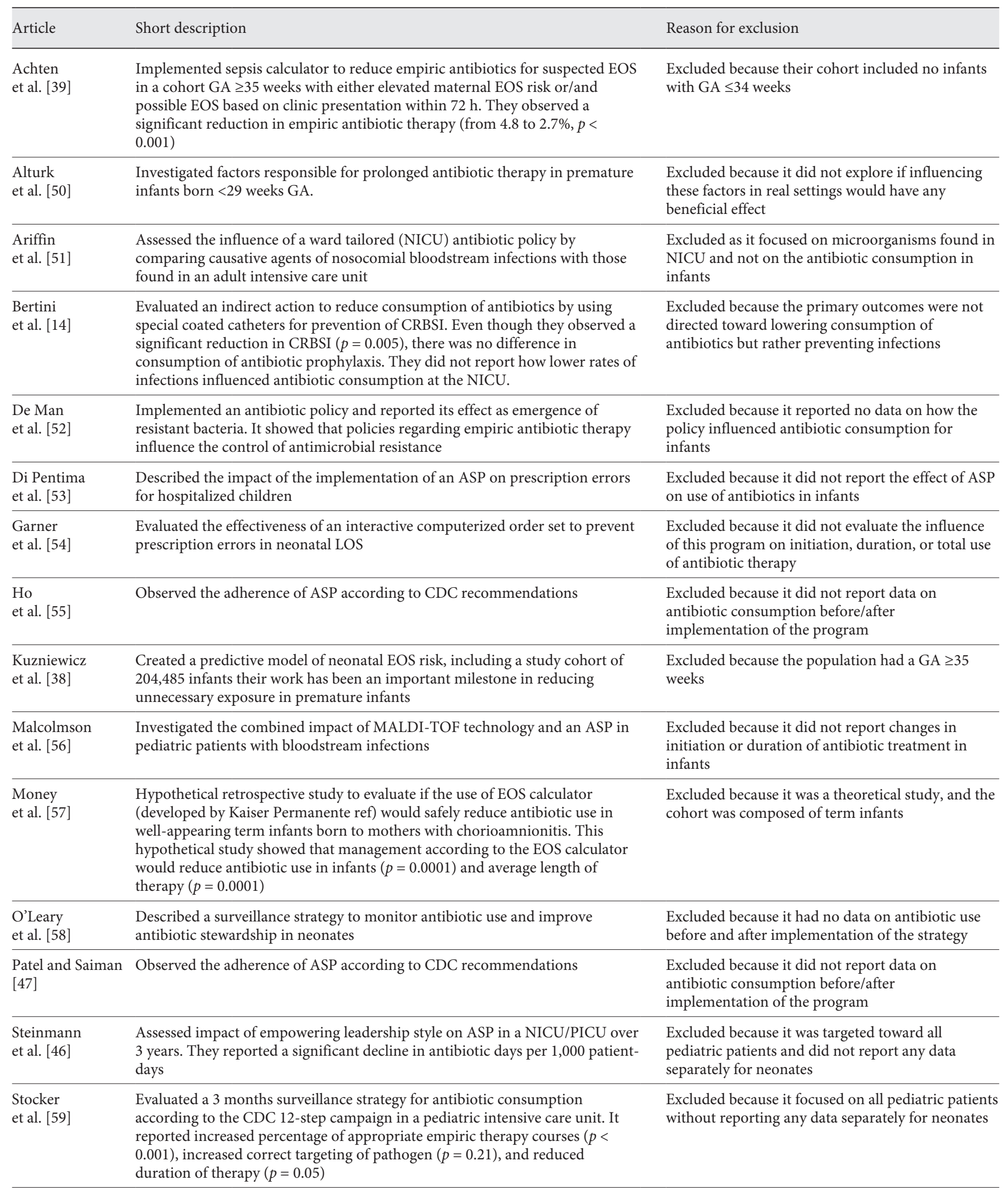


Table 2 (continued)

\begin{tabular}{lll}
\hline Article & Short description & Reason for exclusion \\
\hline $\begin{array}{l}\text { Toltzis } \\
\text { et al. [60] }\end{array}$ & $\begin{array}{l}\text { Focused on the influence of antibiotic rotations in NICU (monthly rotation of } \\
\text { gentamicin, piperacillin-tazobactam, and ceftazidime) on colonization with } \\
\text { resistant microorganism in the infants }\end{array}$ & $\begin{array}{l}\text { Excluded because it focused on microorganisms, } \\
\text { and did not report patent-level data on possible } \\
\text { changes in initiation or duration of antibiotic } \\
\text { treatment }\end{array}$ \\
\hline $\begin{array}{l}\text { Walker } \\
\text { et al. [15] }\end{array}$ & $\begin{array}{l}\text { Showed results of ASP in neonatal surgical patients. This is a specific group of } \\
\text { infants, mostly not premature, in specific situations (antibiotic prophylaxis at } \\
\text { surgical procedures) }\end{array}$ & $\begin{array}{l}\text { Excluded because it targeted a specific group of } \\
\text { infants (neonates with congenital surgical } \\
\text { conditions) with average (IQR) GA 37 (35-39) } \\
\text { weeks }\end{array}$
\end{tabular}

EOS, early-onset sepsis; GA, gestational age; ASP, antibiotic stewardship program; NICU, neonatal intensive care unit; CRBSI, catheter-related bloodstream infections; LOS, late-onset sepsis; CDC, Center for Disease Control and Prevention; MALDI-TOF, matrix-assisted laser desorption/ionizationtime of flight.

Fig. 2. Antibiotic stewardship interventions groups according to common areas of action. (1) Focus on initiation of antibiotic therapy. (2) Focus on shortening duration of antibiotic therapy. (3) Various organizational ASP implementations. There is overlap in the studies implementing various actions. The first 2 areas used actions tailored to NICU patients, while actions from the third area are less specific and could be adapted to health settings in general. ASP, antibiotic stewardship program; NICU, neonatal intensive care unit.

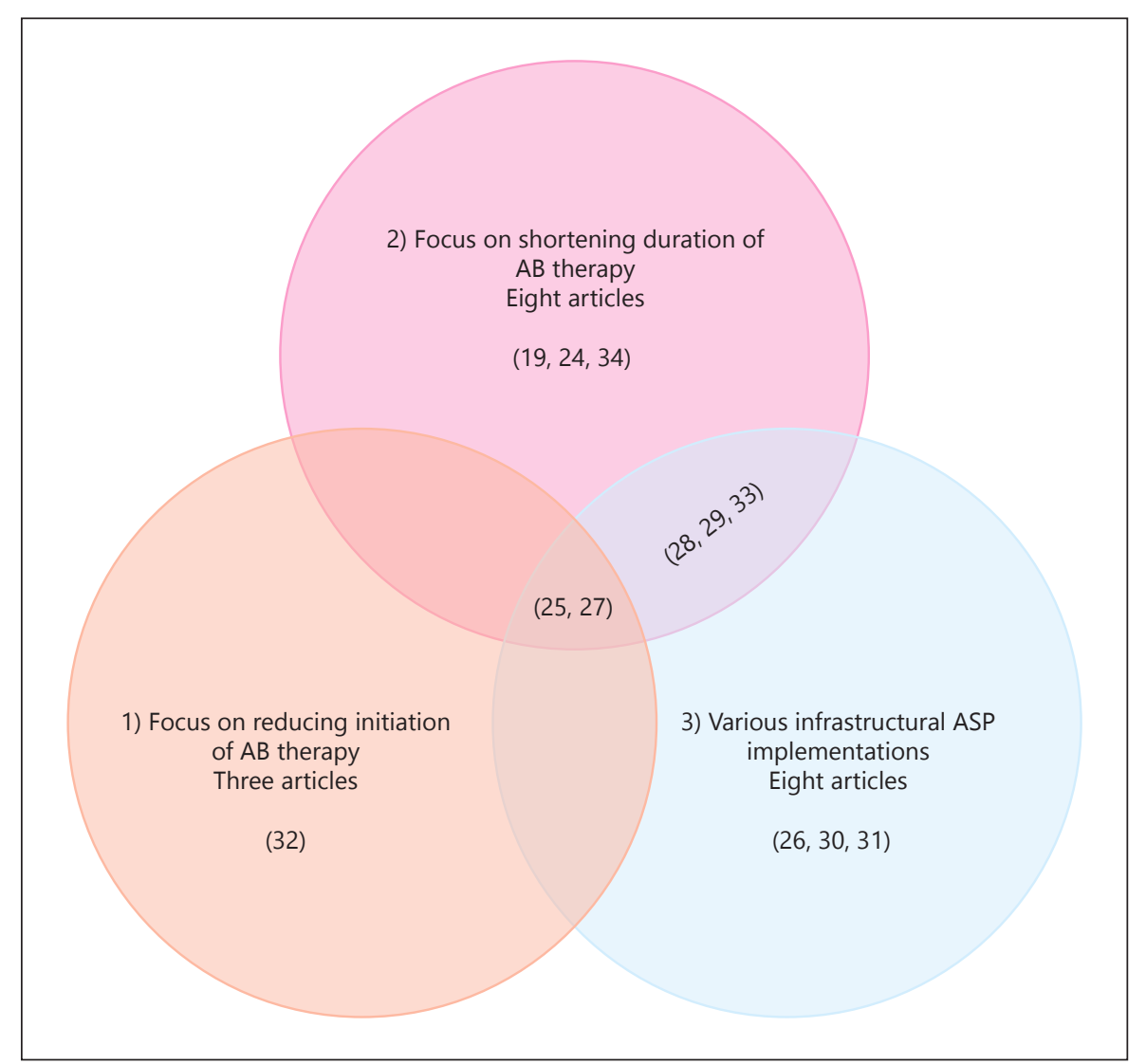

identification of organisms. Kitano et al. and Bhat et al. $[25,27]$ used action from all 3 areas. Bhat et al. [25] aimed to minimize exposure to broad-spectrum antibiotics. A multidisciplinary team created guidelines for management of sepsis, including an algorithm to recognize coagulase-negative blood culture contamination. All staff un- derwent multiple educational and discussion sessions. PCR became routinely used to rapidly identify pathogens from positive blood cultures or cerebrospinal fluid samples [25]. Kitano et al. discussed cases of noncompliance collegiately on a daily basis and made blood culture results available also on weekends and holidays [27]. 


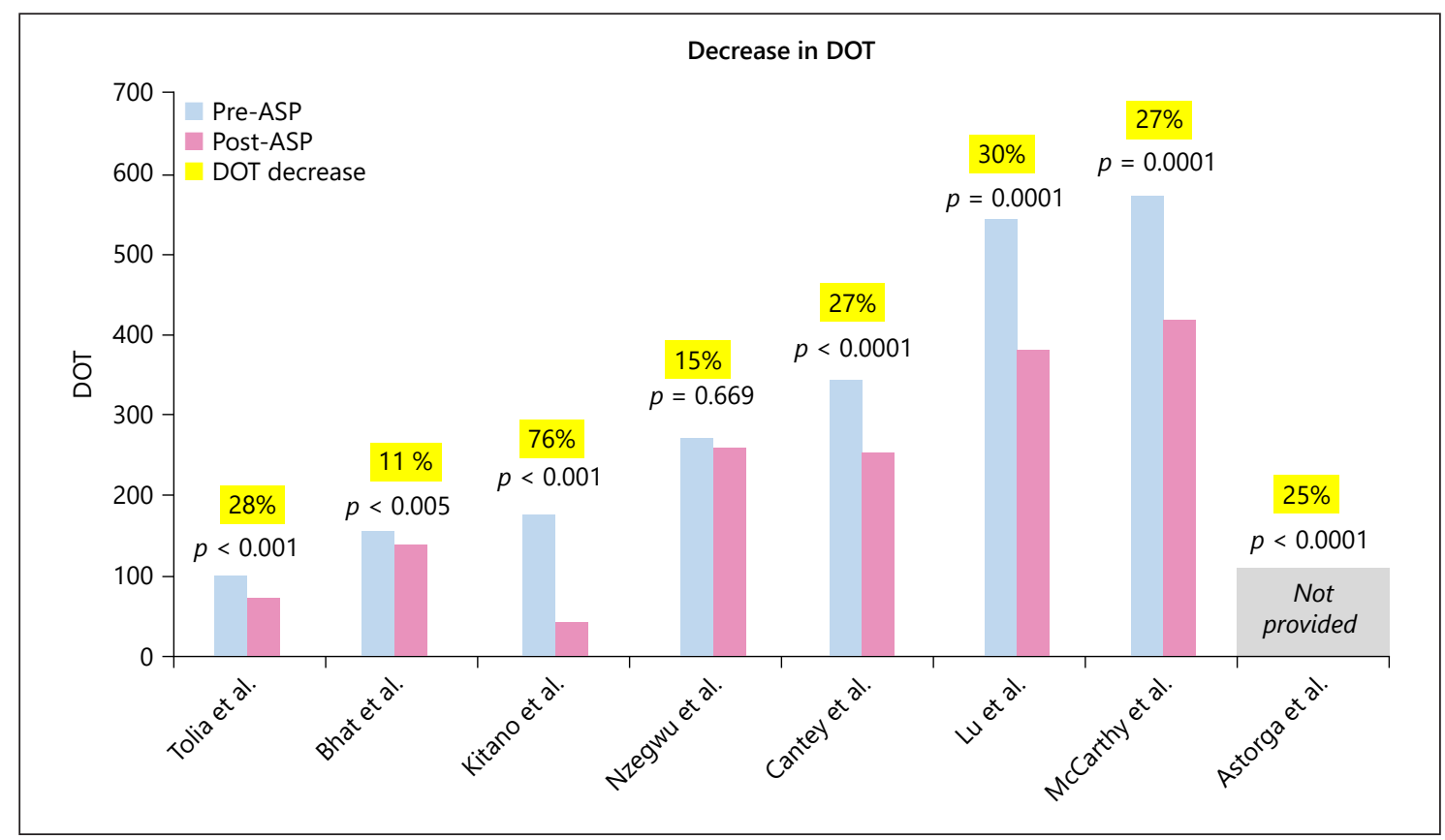

Fig. 3. DOT/1,000 patient-days. Starting with the lowest baseline antibiotic consumption, Tolia et al. [34] achieved a significant reduction from 99.5 to 71.7 DOT patient-days. Bhat et al. [25] achieved reduction of all antibiotic utilization rates from 154.8 to 138.4 DOT. Kitano et al. [27] achieved a decrease from 175.1 to 41.6 DOT. Only one study (Nzegwu et al. [31]) did not find a significant decrease of total antibiotic consumption. Cantey et al. achieved a reduction from 343.2 to 252.2 DOT [19]. Lu et al. [28] achieved a decrease from 543 to 380 DOT. McCarthy et al. [29] reduced antibiotic use from 572 to 417 DOT after second intervention. Astorga et al. [24] was able to achieve a significant $25 \%$ decrease in antibiotic consumption. DOT, days of therapy; ASP, antibiotic stewardship program.

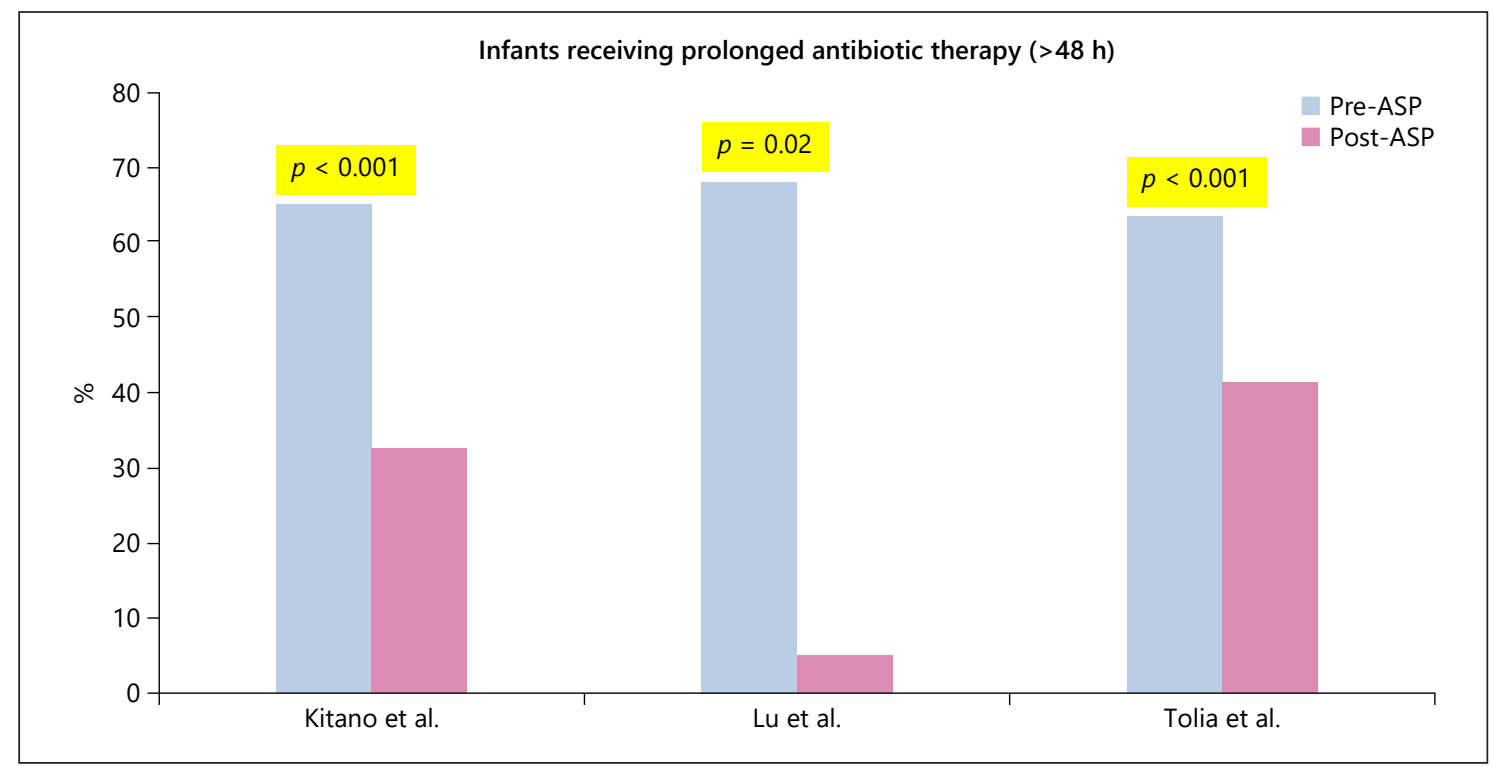

Fig. 4. Percentage of infants receiving prolonged ( $>48 \mathrm{~h}$ ) antibiotic therapy. Kitano et al. [27] achieved a reduction of prolonged antimicrobial treatments from 65 to $32.5 \%$. Lu et al. [28] had an increase in percentage of discontinued antibiotic courses $\leq 48 \mathrm{~h}$ from 32 to $95 \%$. Tolia et al. [34] lowered percentage of infants with $>48 \mathrm{~h}$ of antibiotic exposure from 63.4 to $41.3 \%$. 
Antibiotic Stewardship Articles Grouped according to Common Units of Measurement for Reporting Results Most commonly used units of measurement describing success of ASP in reducing amount of antibiotic consumption were days of therapy/1,000 patient-days (DOT) and defined daily dose/100 patient-days (DDD) [35]. DOT represents the actual number of doses received by the patients and is preferred in pediatrics as dosage is weight- and age-adjusted. DDD gives information of the volume of antibiotic used by a unit. It is easy to obtain (pharmaceutical records) but lacks individual level data.

Papers listed under one of the first two areas of action used individual patient data and mostly expressed their results as DOT, or percentages of infants receiving antibiotic treatment before and after ASP implementation. Two papers using actions from the third area reported their result as DDD.

Days of Therapy/1,000 Patient-Days

Eight out of the 12 studies expressed their result in DOT (Fig. 3) [19, 24, 25, 27-29, 31, 34]. All but one study [31] found significant decrease in total antibiotic consumption.

Additionally, Ting et al. [33] looked at the proportion of infants with negative blood cultures receiving prolonged antibiotic therapy ( $>3$ days) and found a nonsignificant change in inappropriate antibiotic-days $/ 1,000$ DOT courses of therapy with meropenem, cefotaxim, and vancomycin from 1.89 to 1.96 (rate ratio [RR], 1.04 [0.70$1.52]), 3.56$ to 1.73 (RR, 0.49 [0.33-0.71]), and 2.70 to 1.01 (RR, $0.37[0.22-0.60])$, respectively.

\section{Defined Daily Dose/100 Patient-Days}

Two out of the 12 studies used general oriented approaches for their ASP and expressed results as DDD [26, 30]. Jinka et al. [26] observed a nonsignificant reduction of DDD of antibiotic (from 14.47 to 11.47, $p=0.57$ ), but the proportion of babies on antibiotics decreased significantly $(p<0.001)$. They also achieved a significant increase in consumption of first-line antibiotics $(p<0.001)$ and a significant decrease in third generation cephalosporins $(p=0.002)$. The effect of the ASP described by NitschOsuch et al. [30] resulted in a slight increase of DDD (from 28.9 to 30.8). However, they also observed a positively changed antibiotic consumption profile.

Percentage of Infants Starting or Receiving

Prolonged Antibiotic Therapy

Kitano et al. [27] achieved a significant reduction from 55.3 to $20.6 \%(p<0.001)$ infants receiving any antibiotic treatment. Three studies showed decrease in percentage of infants receiving prolonged $(>48 \mathrm{~h}$ ) antibiotic therapy (Fig. 4) $[27,28,34]$. One also showed a decrease in infants with culture-negative sepsis receiving $\geq 5$ days of antibiotics (from 66 baseline to $33 \%$ post-intervention) [28].

The only randomized control trial, performed by Tagare et al. [32], did not report results in units that describe amounts of used antibiotics, but they found no increase in sepsis incidence or mortality in low-risk infants not receiving empiric antibiotics treatment compared to infants receiving 5 days of prophylactic antibiotics (71 infants, sepsis incidence $25.4 \%$, mortality $2.8 \%$ vs. 69 infants, sepsis incidence $31.9 \%$, mortality $2.9 \%$ ), not even in the subgroup of very low birth weight infants (sepsis incidence $42.3 \%$, mortality $3.8 \%$ vs. sepsis incidence $59.3 \%$, mortality $7.4 \%$ for control and intervention groups, respectively).

\section{Discussion}

Several approaches may reduce unnecessary antibiotic exposure. In this systematic review of infants born $\leq 34$ weeks GA, we identified 12 articles describing different ASPs. Due to great heterogeneity in cohorts, implemented actions, and outcome measures, meta-analysis was considered inappropriate. The selected studies also differ in resources and starting point regarding prescribing antibiotics. When the baseline is " 5 days of antibiotics to all premature babies," small efforts are needed for significant improvement. In departments with the most severely ill and fragile neonates and/or where several measures to restrict unnecessary antibiotics have already been implemented, it is harder to see significant positive development [31]. We found reduction in use of antibiotics in studies focusing directly toward reducing initiation or on shortening the duration of antibiotic therapy. Studies focusing solely on general intentions, without specific individual-dependent measures, did not demonstrate the same reduction in consumption. They were, however, able to achieve a reduction in the use of resistance driving broad-specter antibiotics.

There is a general lack of information on ASPs for premature infants, especially those $<34$ weeks GA. Four of the studies selected in our review included exclusively premature infants (GA $<37$ weeks) [25, 32-34], of which 2 studies included only infants born at $<34$ weeks $[25,34]$. Other selected studies included $<50 \%$ infants $<34$ weeks, and results for different stages of prematurity were not always reported separately (details in Table 1). It is, thus, 
not clear if their changes of antibiotic consumption reflect mostly term, late-premature, or more immature infants.

Management of potential sepsis differs between mature and premature infants $[2,36,37]$. There is no online prediction tool (similar to the Kaiser calculator [18, 38, 39]) for infants $<34$ weeks GA, but published protocols similar to Kitano et al. [27] may be useful. This guidance algorithm, successful in reducing initiation of antibiotic therapy, is based on clinical status of mother and infant, sepsis score of the infant, blood culture results, and time progression of symptoms [27]. "Clinical status" and "progression of symptoms" in premature infants are the more challenging parts of such tools, both for initiation and duration of treatment. Several studies showed that an automatic stop of antibiotics after 36-48 h efficiently decreased unnecessary antibiotic exposure in premature infant. Three studies $[24,28,34]$ used this as one of the main or only intervention and found significant reduction in total antibiotic doses on individual levels. Another study implemented automatic stop as their second intervention, after thoroughly revising and troubleshooting their antibiotic prescriptions routines [29] and then significantly reduced antibiotics consumption. One study additionally limited the duration of antibiotic for culturenegative sepsis and pneumonia to 5 days [19]. All studies did, however, require clinical evaluation, some as supplement to the stop order, all for continuous evaluation of need to reconsider treatment.

The quality of the clinician's evaluation is dependent on more than skills. Close monitoring and series of physical examinations may reduce unnecessary initiation of antibiotics [40], but sufficient human and material resources are needed. Furthermore, cooperation with obstetricians is essential for providing exact information of the circumstances of preterm birth and thereby evaluation of risk factors. Proximity and communication with laboratories, their efficacy and ability to provide accurate and suitable biochemical tests (e.g., serial procalcitonin), and fast identification of infectious agents and resistance profile, influence the possibility and the timeline of making decisions $[41,42]$. This may explain some of the variance in use of antibiotics across different NICUs.

We further compared the decrease in DOT in the different studies with the action areas they included in their ASPs (Fig. 3). Kitano et al. and Bhat et al. [25, 27] combined actions from all 3 areas and reported the highest $(76 \%)$ and the lowest (10.6\%) decrease, respectively. Studies combining 2 of the action areas presented medium decrease $(28-30 \%)[28,29,34]$, while the studies fo- cusing on one action area alone achieved a 27 [19], 25 [24], and 15\% [31] decrease in DOT. The 2 studies with the least decrease in DOT had quite low baseline consumption before the reported ASPs. Earlier implemented actions to reduce antibiotic consumption and the differences between the populations (i.e., age and NICU level) are important to consider when evaluating the results (details in Table 1).

Recent research has revealed adverse effect of antibiotic exposure on health of premature infants $[5,43]$. Individual adverse outcomes should be emphasized when deciding on initiating and discontinuing antibiotic treatment. Additionally, antibiotic resistance is a fast-increasing global challenge. Antibiotic therapy in early life disturbs the developing microbiome, increases carriage of antibiotic resistance genes, and could contribute to increased antibiotic resistance in the population [44]. The clinician needs to balance the fear of not providing necessary antibiotics to treat infections with the risk of shortand long-term negative effects. Local customized studies (similar to Tagare et al. [32]) may reduce the fear of the clinicians, of overlooking the need of antibiotics resulting in disastrous effects.

Even though ASPs largely target clinical personnel, it is imperative that also leaders acknowledge the clinical challenges, encourage transparency and nonpunitive culture, and endorse these programs $[45,46]$. The study by Nzegwu et al. [31] also demonstrates the fruits of joint efforts between health authorities and clinicians. They utilized guidelines for design and implementation of a NICU-specific ASP developed by Patel and Saiman [47] which is based on CDC's Get Smart for Healthcare campaign [48]).

\section{Conclusion}

In the reviewed studies, the most successful actions in reducing unnecessary antibiotic exposure in premature infants appeared to be the implementation of multivariable risk assessments and clinical tools developed for decisions on initiation of antibiotic treatment of suspected or potential sepsis, and the use of automatic stop in antibiotic prescriptions. A thorough evaluation of the current state at the NICU also helps identify weak points of antibiotic-prescribing practices and allows for a custom-tailored ASP [29]. In the selected studies, general actions for limiting antibiotic use on the hospital level only were less successful in reducing antibiotic exposure in premature infants but could improve the profile of used antibiotics. 
This lead to the presumption that a locally customized, multifactorial, broad approach, most of all with individual patient-focused outcome measures is preferable.

\section{Limitations}

There are limited studies regarding ASPs for premature infants. Our search string was constructed to find studies using terms such as drug prescription practices or drug utilization. Studies without these terms have been missed by our search. No reports of adverse outcomes of ASPs are documented. Reviewed studies varied in GA of included infants (as most studies encompassed the entire NICU population), in settings, outcome measures, and in the duration of pre- and post-ASP intervention periods. Some variation in assessed quality of included articles was found (online suppl. 3,4). The 11 observational articles achieved scores suggesting high quality ( 7 of more out of 9), while the risk of bias was assessed to be high in the 1 RCT (Jadad score 3 or less). However, the article was not excluded as we did not synthetize any new data, rather summarized and described published findings.

\section{Statement of Ethics}

The paper is exempt for Ethics Committee approval as this is a systematic review using data from published literature and no additional patient data were collected.

\section{Conflict of Interest Statement}

A.D. and S.S.M. are employed by the R\&D division of Tata Consultancy Services Limited (TCS) and declare no conflict of interest. All other authors have no conflict of interest to declare.

\section{Funding Sources}

The Norwegian Research Council (project number 273833) and the Olav Thon Foundation supported the study.

\section{Author Contributions}

F.C.P. and U.R.D. conceived the presented idea. All authors developed the protocol, and P.R., K.H., U.R.D., and O.D.S. performed the literature search. P.R. took the lead in writing the manuscript. All authors provided critical feedback and helped shape the manuscript.

\section{Acknowledgements}

We thank Marie Susanna Isachsen from the University of Oslo library for helping with the protocol for the literature search. This manuscript has been previously published on a preprint server [49].

\section{References}

1 Lucia Hug DS, You D. Levels \& trends in child mortality. UNICEF; 2017 [Cited 2019 Oct 10]

2 Puopolo KM, Benitz WE, Zaoutis TE. Management of neonates born at $\leq 346 / 7$ weeks' gestation with suspected or proven early-onset bacterial sepsis. Pediatrics. 2018 Dec; 142(6):e20182896.

3 Shane AL, Sánchez PJ, Stoll BJ. Neonatal sepsis. Lancet. 2017 Oct 14;390(10104):1770-80.

4 Klingenberg C, Kornelisse RF, Buonocore G, Maier RF, Stocker M. Culture-negative earlyonset neonatal sepsis: at the crossroad between efficient sepsis care and antimicrobial stewardship. Front Pediatr. 2018;6:285.

5 Cotten CM, Taylor S, Stoll B, Goldberg RN, Hansen NI, Sánchez PJ, et al. Prolonged duration of initial empirical antibiotic treatment is associated with increased rates of necrotizing enterocolitis and death for extremely low birth weight infants. Pediatrics. 2009 Jan; 123(1):58-66
6 Kuppala VS, Meinzen-Derr J, Morrow AL, Schibler KR. Prolonged initial empirical antibiotic treatment is associated with adverse outcomes in premature infants. J Pediatr. 2011;159(5):720-5.

7 Ting JY, Synnes A, Roberts A, Deshpandey A, Dow K, Yoon EW, et al. Association between antibiotic use and neonatal mortality and morbidities in very low-birth-weight infants without culture-proven sepsis or necrotizing enterocolitis. JAMA Pediatr. 2016 Dec 1; 170(12):1181-7.

8 Esaiassen E, Fjalstad JW, Juvet LK, van den Anker JN, Klingenberg C. Antibiotic exposure in neonates and early adverse outcomes: a systematic review and meta-analysis. J Antimicrob Chemother. 2017 Jul 1;72(7):1858-70.

9 Gasparrini AJ, Crofts TS, Gibson MK, Tarr PI, Warner BB, Dantas G. Antibiotic perturbation of the preterm infant gut microbiome and resistome. Gut Microbes. 2016 Sep 2;7(5): 443-9.
10 Langdon A, Crook N, Dantas G. The effects of antibiotics on the microbiome throughout development and alternative approaches for therapeutic modulation. Genome Med. 2016; $8(1): 39$.

11 MacDougall C, Polk RE. Antimicrobial stewardship programs in health care systems. Clin Microbiol Rev. 2005 Oct;18(4):638-56.

12 Armstrong GL, Conn LA, Pinner RW. Trends in infectious disease mortality in the United States during the 20th century. JAMA. 1999 Jan 6;281(1):61-6.

13 Superbugs threaten hospital patients. Center for Dissease Control and Prevention. Prevention.Atlanta, GA: March 2016 [Cited 2019 Dec 10]. Available from: https://www.cdc.gov/media/releases/2016/p0303-superbugs.html.

14 Bertini G, Elia S, Ceciarini F, Dani C. Reduction of catheter-related bloodstream infections in preterm infants by the use of catheters with the AgION antimicrobial system. Early Hum Dev. 2013;89(1):21-5. 
15 Walker S, Datta A, Massoumi RL, Gross ER, Uhing M, Arca MJ. Antibiotic stewardship in the newborn surgical patient: a quality improvement project in the neonatal intensive care unit. Surgery. 2017 Dec;162(6):1295303.

16 World Health Organization. Infection prevention and control [Cited 2019 May 31]. Available from: https://www.who.int/gpsc/ ipc/en/.

17 Baur D, Gladstone BP, Burkert F, Carrara E, Foschi F, Döbele S, et al. Effect of antibiotic stewardship on the incidence of infection and colonisation with antibiotic-resistant bacteria and Clostridium difficile infection: a systematic review and meta-analysis. Lancet Infect Dis. 2017 Sep;17(9):990-1001.

18 Achten NB, Klingenberg C, Benitz WE, Stocker M, Schlapbach LJ, Giannoni E, et al. Association of use of the neonatal early-onset sepsis calculator with reduction in antibiotic therapy and safety: a systematic review and meta-analysis. JAMA Pediatr. 2019;173(11): 1032-40.

19 Cantey JB, Wozniak PS, Pruszynski JE, Sánchez PJ. Reducing unnecessary antibiotic use in the neonatal intensive care unit (SCOUT): a prospective interrupted time-series study. Lancet Infect Dis. 2016 Oct;16(10):1178-84.

20 Flannery DD, Ross RK, Mukhopadhyay S, Tribble AC, Puopolo KM, GerberTemporal Trends JS, et al. Temporal trends and center variation in early antibiotic use among premature infants. JAMA Netw Open. 2018;1(1): e180164-64.

21 Norwegian Neonatal Network database (NNK). Nasjonalt Servicemiljø for medisinske kvalitetsregistre. [Cited 2019 Aug 27]. Available from: http://www.kvalitetsregistre. no/resultater/skade-og-inten-sivbehandling/ norsk-nyfoedtmedisinsk-kvalitetsregister/.

22 Broadfoot M. A delicate balance. Science. 2018 Apr 6;360(6384):18-20.

23 Fenton TR. A new growth chart for preterm babies: Babson and Benda's chart updated with recent data and a new format. BMC Pediatr. 2003 Dec 16;3:13.

24 Astorga MC, Piscitello KJ, Menda N, Ebert AM, Ebert SC, Porte MA, et al. Antibiotic stewardship in the neonatal intensive care unit: effects of an automatic 48-hour antibiotic stop order on antibiotic use. J Pediatric Infect Dis Soc. 2019 Sep 25;8(4):310-6.

25 Bhat R, Custodio H, McCurley C, Whitehurst $\mathrm{R}$, Gulati R, Jha OP, et al. Reducing antibiotic utilization rate in preterm infants: a quality improvement initiative. J Perinatol. 2018 Apr; 38(4):421-9.

26 Jinka DR, Gandra S, Alvarez-Uria G, Torre N, Tadepalli D, Nayakanti RR. Impact of antibiotic policy on antibiotic consumption in a neonatal intensive care unit in India. Indian Pediatr. 2017 Sep 15;54(9):739-41.
27 Kitano T, Takagi K, Arai I, Yasuhara H, Ebisu $\mathrm{R}$, Ohgitani A, et al. A simple and feasible antimicrobial stewardship program in a neonatal intensive care unit of a Japanese community hospital. J Infect Chemother. 2019 Nov; 25(11):860-5.

$28 \mathrm{Lu}$ C, Liu Q, Yuan H, Wang L. Implementation of the smart use of antibiotics program to reduce unnecessary antibiotic use in a neonatal ICU: a prospective interrupted time-series study in a developing country. Crit Care Med. 2019 Jan;47(1):e1-7.

29 McCarthy KN, Hawke A, Dempsey EM. Antimicrobial stewardship in the neonatal unit reduces antibiotic exposure. Acta Paediatr. 2018 Oct;107(10):1716-21.

30 Nitsch-Osuch A, Kurpas D, Kuchar E, Zycińska K, Zielonka T, Wardyn K. Antibiotic consumption pattern in the neonatal special care unit before and after implementation of the hospital's antibiotic policy. Adv Exp Med Biol. 2015;835:45-51.

31 Nzegwu NI, Rychalsky MR, Nallu LA, Song X, Deng Y, Natusch AM, et al. Implementation of an antimicrobial stewardship program in a neonatal intensive care unit. Infect Control Hosp Epidemiol. 2017 Oct;38(10):1137-43.

32 Tagare A, Kadam S, Vaidya U, Pandit A. Routine antibiotic use in preterm neonates: a randomised controlled trial. J Hosp Infect. 2010; 74(4):332-6.

33 Ting JY, Paquette V, Ng K, Lisonkova S, Hait $\mathrm{V}$, Shivanada S, et al. Reduction of inappropriate antimicrobial prescriptions in a tertiary neonatal intensive care unit after antimicrobial stewardship care bundle implementation. Pediatr Infect Dis J. 2019 Jan;38(1):54-9.

34 Tolia VN, Desai S, Qin H, Rayburn PD, Poon G, Murthy K, et al. Implementation of an automatic stop order and initial antibiotic exposure in very low birth weight infants. Am J Perinatol. 2017 Jan;34(2):105-10.

35 Guillot J, Lebel D, Roy H, Ovetchkine P, Bussières J-F. Usefulness of defined daily dose and days of therapy in pediatrics and obstetrics-gynecology: a comparative analysis of antifungal drugs (2000-2001, 2005-2006, and 2010-2011). J Pediatr Pharmacol Ther. 2014; 19(3):196-201.

36 Puopolo KM. New sepsis guidance addresses epidemiology, microbiology, recommended empiric treatment AAP News 2018. [Cited 2020 Jan 13]. Available from: https://www. aappublications.org/news/2018/11/19/ sepsis 111918 ? utm_source $=$ TrendMD \& utm_medium=TrendMD\& utm_campaign= AAPNews_TrendMD_0.

37 Puopolo KM, Benitz WE, Zaoutis TE. Management of neonates born at $\leq 346 / 7$ weeks' gestation with suspected or proven early-onset bacterial sepsis. Pediatrics. 2018 Dec; 142(6):e20182896.

38 Kuzniewicz MW, Puopolo KM, Fischer A, Walsh EM, Li S, Newman TB, et al. A quantitative, risk-based approach to the management of neonatal early-onset sepsis. JAMA Pediatr. 2017 Apr 1;171(4):365-71.
39 Achten NB, Dorigo-Zetsma JW, van der Linden PD, van Brakel M, Plötz FB. Sepsis calculator implementation reduces empiric antibiotics for suspected early-onset sepsis. Eur J Pediatr. 2018 May;177(5):741-6.

40 Berardi A, Buffagni AM, Rossi C, Vaccina E, Cattelani C, Gambini L, et al. Serial physical examinations, a simple and reliable tool for managing neonates at risk for early-onset sepsis. World J Clin Pediatr. 2016 Nov 8;5(4):358-64.

41 Romaniszyn D, Różańska A, WójkowskaMach J, Chmielarczyk A, Pobiega M, Adamski $\mathrm{P}$, et al. Epidemiology, antibiotic consumption and molecular characterisation of Staphylococcus aureus infections: data from the Polish Neonatology Surveillance Network, 20092012. BMC Infect Dis. 2015 Apr 1;15:169.

42 Paul SP, Caplan EM, Morgan HA, Turner PC Barriers to implementing the NICE guidelines for early-onset neonatal infection: crosssectional survey of neonatal blood culture reporting by laboratories in the UK. J Hosp Infect. 2018 Apr;98(4):425-8.

43 Alexander VN, Northrup V, Bizzarro MJ. Antibiotic exposure in the newborn intensive care unit and the risk of necrotizing enterocolitis. J Pediatr. 2011 Sep;159(3):392-7.

44 Gasparrini AJ, Wang B, Sun X, Kennedy EA, Hernandez-Leyva A, Ndao IM, et al. Persistent metagenomic signatures of early-life hospitalization and antibiotic treatment in the infant gut microbiota and resistome. Nat Microbiol. 2019 Dec;4(12):2285-97.

45 Lawrence KL, Kollef MH. Antimicrobial stewardship in the intensive care unit: advances and obstacles. Am J Respir Crit Care Med. 2009 Mar 15;179(6):434-8.

46 Steinmann KE, Lehnick D, Buettcher $M$, Schwendener-Scholl K, Daetwyler K, Fontana $\mathrm{M}$, et al. Impact of empowering leadership on antimicrobial stewardship: a single center study in a neonatal and pediatric intensive care unit and a literature review. Front Pediatr. 2018;6:294.

47 Patel SJ, Saiman L. Principles and strategies of antimicrobial stewardship in the neonatal intensive care unit. Semin Perinatol. 2012 Dec; 36(6):431-6.

48 Centers for Disease Control. Get smart for healthcare. 2017. [Cited 2020 Jan 13]. Available from: https://www.cdc.gov/getsmart/ healthcare/.

49 Rajar P, Saugstad OD, Berild D, Dutta A, Greisen G, Lausten-Thomsen U, et al. Antibiotic stewardship in premature infants: a systematic review. medRxiv. 2020.

50 Alturk MR, Baier RJ. Patient and prescriber factors and the prolongation of antibiotics after birth in infants less than 29 weeks. J Matern Fetal Neonatal Med. 2018 Jul;31(13):17201726. doi: 10.1080/14767058.2017.1326896.

51 Ariffin N, Hasan H, Ramli N, Ibrahim NR, Taib F, Rahman AA, et al. Comparison of antimicrobial resistance in neonatal and adult intensive care units in a tertiary teaching hospital. Am J Infect Control. 2012 Aug;40(6): 572-5. doi: 10.1016/j.ajic.2012.02.032.
Antibiotic Stewardship in Premature Infants
Neonatology 2020;117:673-686

DOI: $10.1159 / 000511710$ 
52 de Man P, Verhoeven BA, Verbrugh HA, Vos MC, van den Anker JN. An antibiotic policy to prevent emergence of resistant bacilli. Lancet. 2000 Mar 18355;(9208):973-8. doi: 10.1016/s0140-6736(00)90015-1.

53 Di Pentima MC, Chan S. Impact of antimicrobial stewardship program on vancomycin use in a pediatric teaching hospital. Pediatr Infect Dis J. 2010 Aug;29(8):707-11. doi: 10.1097/ INF.0b013e3181d683f8.

54 Garner SS, Cox TH, Hill EG, Irving MG, Bissinger RL, Annibale DJ. Prospective, controlled study of an intervention to reduce errors in neonatal antibiotic orders. J Perinatol. 2015 Aug; 35(8):631-5. doi: 10.1038/ jp.2015.20.
55 Ho T, Buus-Frank ME, Edwards EM, Morrow KA, Ferrelli K, Srinivasan A, et al. Adherence of Newborn-Specific Antibiotic Stewardship Programs to CDC Recommendations. Pediatrics. 2018 Dec; 142(6):e20174322. doi: 10.1542/peds.2017-4322.

56 Malcolmson C, Ng K, Hughes S, Kissoon N, Schina J, Tilley PA, et al. Impact of MatrixAssisted Laser Desorption and Ionization Time-of-Flight and Antimicrobial Stewardship Intervention on Treatment of Bloodstream Infections in Hospitalized Children. J Pediatric Infect Dis Soc. 2017 Jun 16;(2):178186. doi: 10.1093/jpids/piw033.

57 Money N, Newman J, Demissie S, Roth P, Blau J. Anti-microbial stewardship: antibiotic use in well-appearing term neonates born to mothers with chorioamnionitis. J Perinatol. 2017 Dec;37(12):1304-1309. doi: 10.1038/ jp.2017.137.
58 O'Leary EN, van Santen KL, Edwards EM, Braun D, Buus-Frank ME, Edwards JR, et al. Using NHSN's Antimicrobial Use Option to Monitor and Improve Antibiotic Stewardship in Neonates. Hosp Pediatr. 2019 May; 9(5):340-347. doi: 10.1542/hpeds.2018-0265.

59 Stocker M, Ferrao E, Banya W, Cheong J, Macrae D, Furck A. Antibiotic surveillance on a paediatric intensive care unit: easy attainable strategy at low costs and resources. BMC Pediatr. 2012 Dec;12:196. doi: 10.1186/14712431-12-196.

60 Toltzis P, Dul MJ, Hoyen C, Salvator A, Walsh $\mathrm{M}$, Zetts L, et al. The effect of antibiotic rotation on colonization with antibiotic-resistant bacilli in a neonatal intensive care unit. Pediatrics. 2002 Oct;110(4):707-11. doi: 10.1542/ peds.110.4.707. 